\title{
ARTICLE OPEN \\ Coat-and-print patterning of silver nanowires for flexible and transparent electronics
}

\author{
Weiwei Li iD ${ }^{1 *}$, Azat Meredov ${ }^{1}$ and Atif Shamim ${ }^{1}$
}

\begin{abstract}
Silver nanowires (Ag NWs) possess excellent optoelectronic properties, which have led to many technology-focused applications of transparent and flexible electronics. Many of these applications require patterning of Ag NWs into desired shapes, for which maskbased and printing-based techniques have been developed and widely used. However, there are still several limitations associated to these techniques. These limitations, such as complicated patterning procedures, limited patterning area, and compromised optical transparency, hamper the efficient fabrication of high-performance Ag NW patterns. Here, we propose a coat-and-print approach for effectively patterning Ag NWs. We printed a polymer-based ink on the spin-coated Ag NW films. The ink acts as a protective layer to help remove excess Ag NWs from the substrate and then dissolves itself into an organic solvent. In this way, we can take advantage of both coating-based techniques (lead to Ag NWs with high transparency) and printing-based techniques (efficiently pattern diverse shapes). The resultant Ag NW patterns exhibit comparable conductivity (sheet resistance: 7.1 to 30 Ohm/ sq) and transparency (transmittance: 84 to $95 \%$ at $\lambda=550 \mathrm{~nm}$ ) to those made by conventional coating methods. In addition, the patterned Ag NWs exhibit robust mechanical stability and reliability, surviving extensive bending and peeling tests. Due to higher conductivity, efficient patterning ability and inherent transparency, this material system and application method is highly suitable for transparent and flexible electronics. As a proof of concept, this research demonstrates a wide-band antenna, operating in the $\mathrm{mm}$-wave range that includes the $5 \mathrm{G}$ communication band. The proposed antenna exhibits a wide bandwidth of $26 \mathrm{GHz}$ (from $17.9 \mathrm{GHz}$ to $44 \mathrm{GHz}$ ), robust return loss under 1000 cyclic bending (bending radius of $3.5 \mathrm{~mm}$ ), and decent transparency over the entire visible wavelength $(86.8 \%$ transmittance at $\lambda=550 \mathrm{~nm})$. This work's promising results indicate that this method can be adapted for roll-to-roll manufacturing to efficiently produce patterned and optically transparent devices.
\end{abstract}

npj Flexible Electronics (2019)3:19; https://doi.org/10.1038/s41528-019-0063-3

\section{INTRODUCTION}

Transparent, flexible electronics have gained emerging attention in a variety of fields, including optoelectronics, energy storages, sensors, and light emitting displays. ${ }^{1-5}$ However, conventional conductors, such as indium tin oxide (ITO), have long suffered from high processing cost and lack of flexibility. Motivated by this issue, researchers have developed many ITO alternatives by using conductive materials with decreasing dimensions, including nanoscale metal mesh, ${ }^{6-10}$ conductive polymer, ${ }^{11,12}$ metal nanowires, $^{13-15}$ graphene, ${ }^{16-21}$ and carbon nanotubes. ${ }^{22-24}$ Among these alternatives, silver nanowires (Ag NWs) are particularly attractive due to their excellent electrical conductivity and mechanical flexibility. ${ }^{25-29}$ In the past decade, tremendous efforts have been made to synthesize Ag NWs with high aspect ratio which is critical to the performance enhancement of Ag NWsbased devices. Thus far, various synthesis methods have been proposed, such as low-temperature, ${ }^{28}$ gentle reaction, ${ }^{25}$ multistep growth, ${ }^{30,31}$ and hydrothermal reduction, ${ }^{32}$ leading to ultrahigh aspect ratio of Ag NWs. Moreover, the resultant flexible Ag NW films have simultaneously demonstrated remarkable transparency and low sheet resistance, ${ }^{33-36}$ both of which are comparable and even superior to the ITO films.

Many of the Ag NW-based devices, such as electrodes, circuits, sensors, and thin film transistors, require Ag NW patterns on desired substrates. ${ }^{37-39}$ For this purpose, two types of techniques (i.e., mask-based and printing-based) are proposed and widely used for the patterning of Ag NWs. However, there are still several limitations to both methods. The mask-based patterning techniques, such as photolithography ${ }^{40}$, stencil screen, ${ }^{39}$ wet/dry transfer, ${ }^{37}$ and ultraviolet/ozone $\left(\mathrm{UV} / \mathrm{O}_{3}\right)$ irradiation, ${ }^{41-43}$ are capable of producing highly transparent patterns, as this type of techniques can take advantage of state-of-the-art Ag NWs with high aspect ratio. However, mask use typically requires complex and high-cost processes that yield limited patterning area, as well as low patterning efficiency. By contrast, direct printing technologies offers roll-to-roll compatibility ${ }^{44-47}$ by which large-area, arbitrary conductive patterns have been achieved in cost-effective and time-effective way. ${ }^{48-50}$ However, the printing-based techniques only work for high-concentration inks or short Ag NWs (i.e., NWs with aspect ratios of less than 50). ${ }^{44,47}$ Consequently, the printed Ag NW patterns perform poorly in optical properties as compared to the mask-based patterns. ${ }^{42-44,46,51}$ Therefore, the efficient manufacturing of high-performance Ag NW patterns, on which the success of Ag NW-based devices heavily hinges, remains challenging.

In this work, we introduce a coat-and-print method for manufacturing Ag NW patterns that offers a solution to the challenges mentioned above. Instead of directly printing Ag NWs, we pattern a polymer-based ink on the spin-coated Ag NW films with the aid of inkjet printing. This polymeric pattern acts as a protective layer that can help remove the excess Ag NWs from the substrate and then dissolves in an organic solvent. We show that the resultant Ag NW patterns can be as conductive and transparent as the coating-based or mask-based patterns and be 
(a)

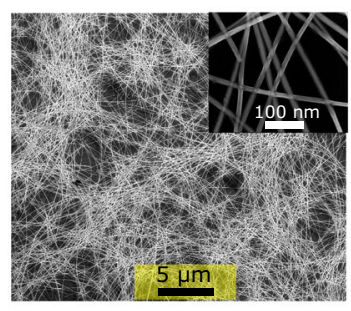

(b)

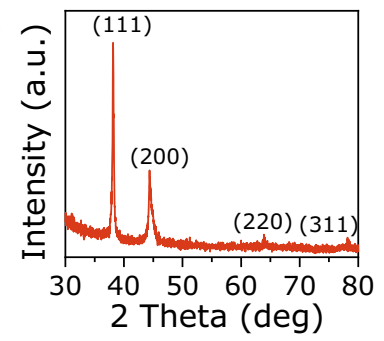

(c)
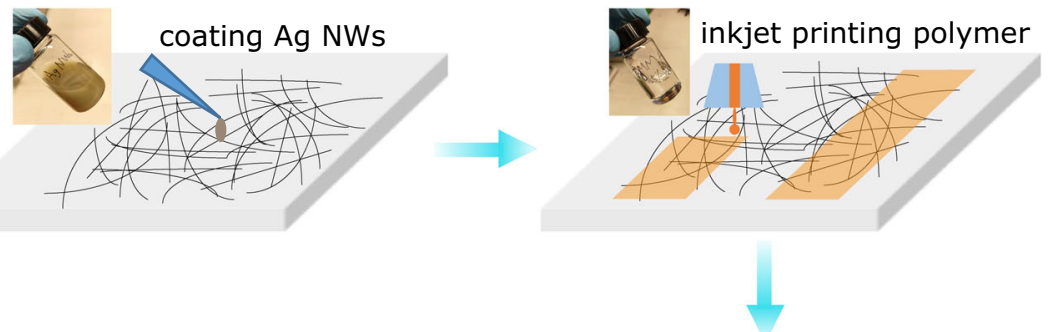

polymer removing

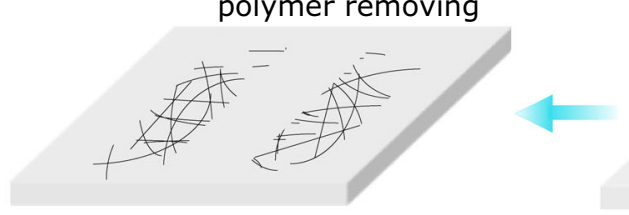

excess Ag NWs removing (d)

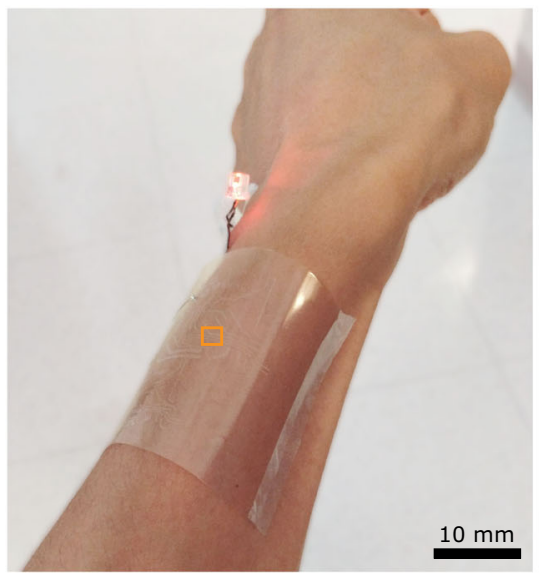

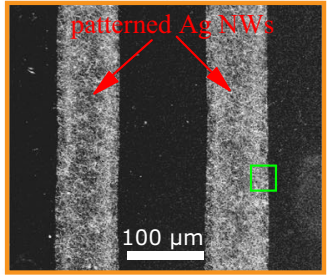

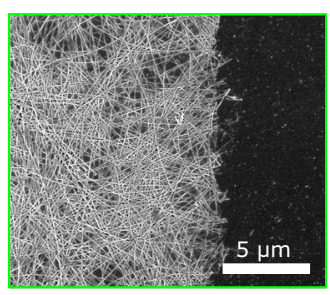

(e) 100

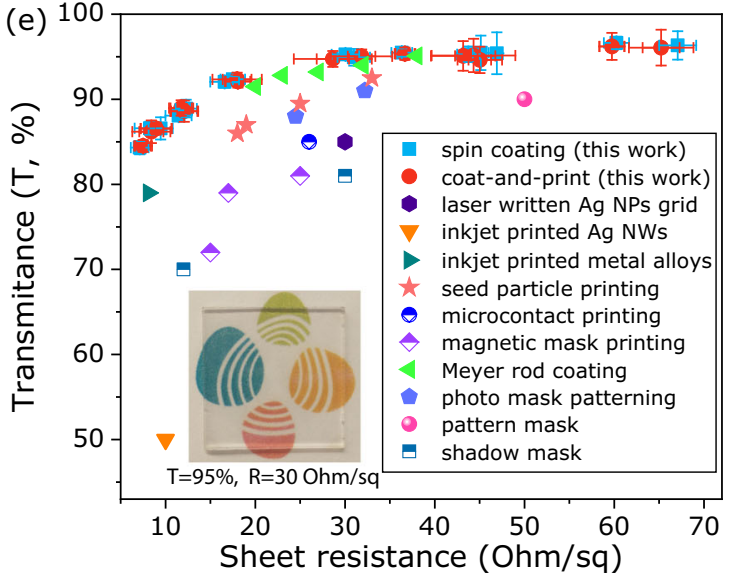

Fig. 1 a SEM image and inset of (a) is a magnified SEM image of the Ag NWs. b XRD pattern of the synthesized Ag NWs. c Schematic diagram of the coat-and-print patterning procedures; (i) depositing Ag NW by drop coating or spin coating on clean substrates to form uniform Ag NW layer; (ii) inkjet printing of polymer ink to form desired patterns; (iii) removing excess Ag NWs with wet tissues or tapes; and (iv) removing polymer by dissolving in organic solvents. Insets are digital photographs of the Ag NW ink and the PMMA ink used in the patterning process. d LED light connected to the patterned transparent Ag NW circuits. The right panel displays the SEM images of the patterned Ag NW lines. e The transmittance $(T$, at $\lambda=550 \mathrm{~nm})$ as a function of sheet resistance $(R)$ of the Ag NW films fabricated by coating, and coat-and-print technique, as well as previously developed methods to prepare transparent electrodes. Inset depicts the photograph of one representative Ag NW patterns on glass $(T=95 \%, R=30 \mathrm{Ohm} / \mathrm{sq})$ in front of KAUST logo.

fabricated as efficiently as the inkjet-printed ones. We further pattern the Ag NWs on a flexible substrate capable of sustaining thousands of bending and peeling cycles. The combination of our sample's strong electrical performances and mechanical durability using the coat-and-print technique makes them particularly promising for the next-generation of flexible and transparent electronics. As a proof of concept, this research demonstrated a flexible and transparent antenna operating in the $\mathrm{mm}$-wave range, including the $5 \mathrm{G}$ communication band.

\section{RESULTS}

The Ag NWs used for the patterning process had a average diameter of $27 \pm 9 \mathrm{~nm}$, and length of $31 \pm 7 \mu \mathrm{m}$, which was confirmed by the scanning electron microscope (SEM) images presented in Fig. 1a and Fig. S1 in the Supporting Information. The typical X-ray diffraction (XRD) pattern of the Ag NWs exhibited four diffraction peaks indexed to the face-centered cubic Ag without any impurities. In addition, the intensity of peak [111] is much higher than those of [200], [220], and [311] due to the high aspect ratio of the Ag NWs, which is ideal for our coat-and-print method to build highly transparent and conductive Ag NW patterns. However, such long Ag NWs are hard to print with an inkjet printing technique, as the nozzle diameter $(\sim 21.5 \mu \mathrm{m})$ is smaller than the length of $\mathrm{Ag} \mathrm{NWs,} \mathrm{which} \mathrm{may} \mathrm{cause} \mathrm{nozzle}$ clogging.

A schematic of the freeform coat-and-print patterning process is presented in Fig. 1c. First, we deposited the Ag NW dispersion onto clean substrates, such as glass and plastic polymers to form a uniform and dense Ag NW network by simply drop casting or spin coating. Such coating can maximize the performances of Ag NW films in both low sheet resistance and high optical transparency. Second, we printed a polymeric, protective layer onto the coated Ag NW films using an inkjet printing technique. Here, we developed a cheap poly(methyl methacrylate) (PMMA) ink (see detailed ink formula and printing process in Experimental section) because (i) strongly adheres to the substrates; (ii) it is not dissolvable in water for the following wet wiping process; and (iii) it is readily removable by an organic solvent. Third, the printed protective layer was baked at $110^{\circ} \mathrm{C}$ in an oven for $5 \mathrm{~min}$ to evaporate solvents and form a uniform PMMA layer (Fig. S2a). The unprotected Ag NWs could be easily erased from the substrate by wiping with a wet tissue or peeling with tape, leaving the Ag NWs on PMMA-patterned regions (Fig. S2b). Finally, we removed the 
(a)

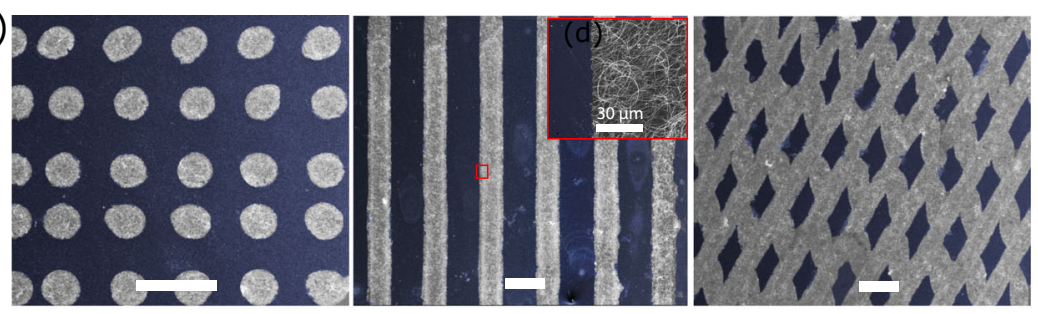

(b)

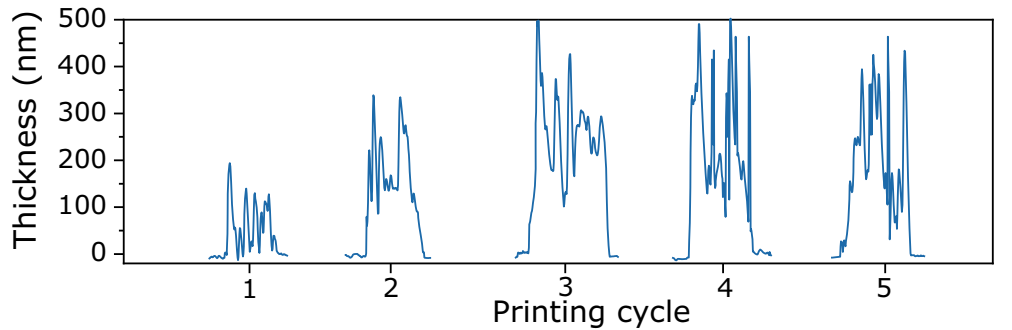

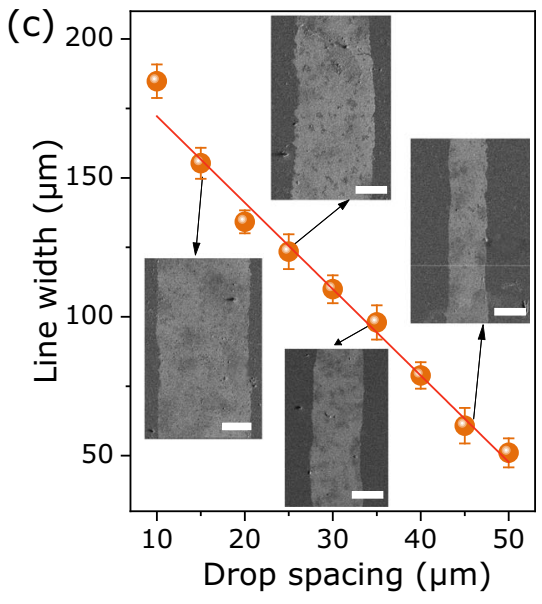

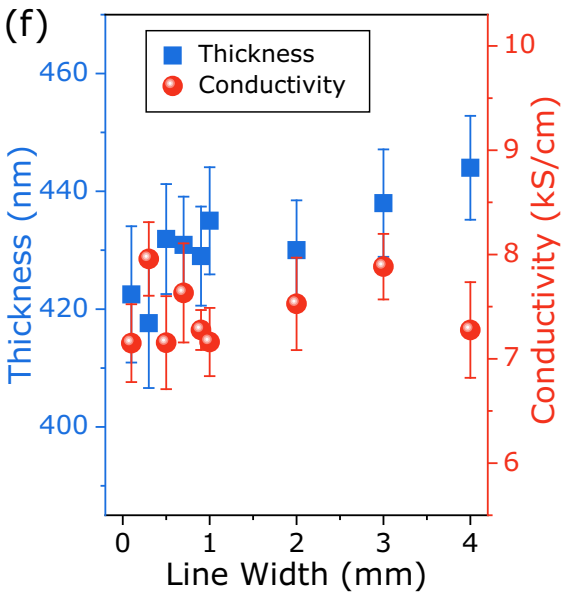

Fig. 2 a SEM images of the patterned dots (diameter $60 \mu \mathrm{m}$ ), lines ( $80 \mu \mathrm{m}$ in spacing), and cross lines ( $60 \mu \mathrm{m}$ in width). All the unmarked scale bars are $100 \mu \mathrm{m}$. $\mathbf{b}$ The line thickness of the patterned Ag NWs as a function of the printing passes. $\mathbf{c}$ The line width plotted as a function of the interdrop spacing. Insets are SEM images of the patterned Ag NW lines. Scale bars are $100 \mu \mathrm{m}$. $\mathbf{d}$ Measured resistance with different line length and various width from three separate Ag NW patterns. e Calculated sheet resistance plotted as a function of line width for the patterned Ag NW lines. $\mathbf{f}$ Thickness and calculated electrical conductivity as a function of line width for the patterned Ag NW lines.

PMMA layer by immersing the sample into acetone for $5 \mathrm{~min}^{52}$ and completed the patterning processes (Fig. S2c). What should be noted is that the etching time to dissolve the PMMA layer highly depends on the solubility of PMMA in organic solvents and the bath temperature. We can decrease the etching time to less than $30 \mathrm{~s}$ in anisole and $\mathrm{N}, \mathrm{N}$-dimethylformamide (DMF) bath at $60^{\circ} \mathrm{C}$ (Fig. S2d, e). Figure 1d demonstrates typical Ag NW patterns on a flexible polyethylene terephthalate (PET) substrate, where fine, micro-scale structures are designed and patterned on the $\mathrm{Ag}$ NW networks as displayed in the magnified SEM images. These patterning steps can be easily repeated on other substrates, such as four-inch fused silica glass, $50 \times 50 \mathrm{~mm}^{2}$ flexible PET and $50 \times$ $50 \mathrm{~mm}^{2}$ Kapton film, as depicted in Fig. S3 in the Supporting Information, which demonstrates the patterned KAUST logo, letters, serpentine lines, straight lines, capacitors, circles, stars, and other complex drawings with clear edges and boundaries.

We further studied the electrical and optical performance of various coated and patterned Ag NW films, which were controlled by changing the coating cycles (one to five cycles) and Ag NW concentration $(0.5-1.5 \mathrm{mg} / \mathrm{mL})$. The transmittance of the Ag NW films changed from 84.2 to $95.6 \%$ when sheet resistance increased from 7.1 to $36 \mathrm{Ohm} / \mathrm{sq}$ (square markers in Fig. 1e). As illustrated in Fig. 1e, our coat-and-print samples (red markers) are nearly identical to the originally coated ones (blue markers) in both conductivity and transmittance, regardless of the Ag NW films used. This result implies that the printed PMMA has been removed during the patterning process and the extra processing in our coat-and-print method causes no noticeable performance degradation to the Ag NW films, indicating the stability and reliability of the proposed patterning technique. Moreover, to achieve the same sheet resistance, our coat-and-print Ag NW patterns feature much higher optical transmittance than the patterns by previously reported printing-based methods (Fig. 1e). The reason is attributed to the ultrahigh aspect ratio of the Ag NWs $(\sim 1000)$ used in this work, whereas only short nanowires (aspect ratio $<50$ ) can be used in the reported inkjet printing process. ${ }^{44,47}$ Moreover, Ag NW films patterned by our method demonstrates comparable electrical and optical performance to the reported methods, such as photo mask patterning, ${ }^{43}$ pattern mask patterning, ${ }^{42}$ inkjet printing ${ }_{r}^{44}$ micro-contact printing ${ }^{41}$ shadow mask patterning, ${ }^{53}$ and magnetic mask printing, ${ }^{54}$ as well as other transparent electrodes, such as seed particle printing of metal alloys ${ }^{55}$ and laser writing of $\mathrm{Ag}$ nanoparticles grid. ${ }^{10}$ We thus suggest that our coat-and-print method can take advantage of both coating-based techniques (can lead to patterns with high conductivity and transparency) and printing-based techniques (can efficiently pattern diverse shapes). 

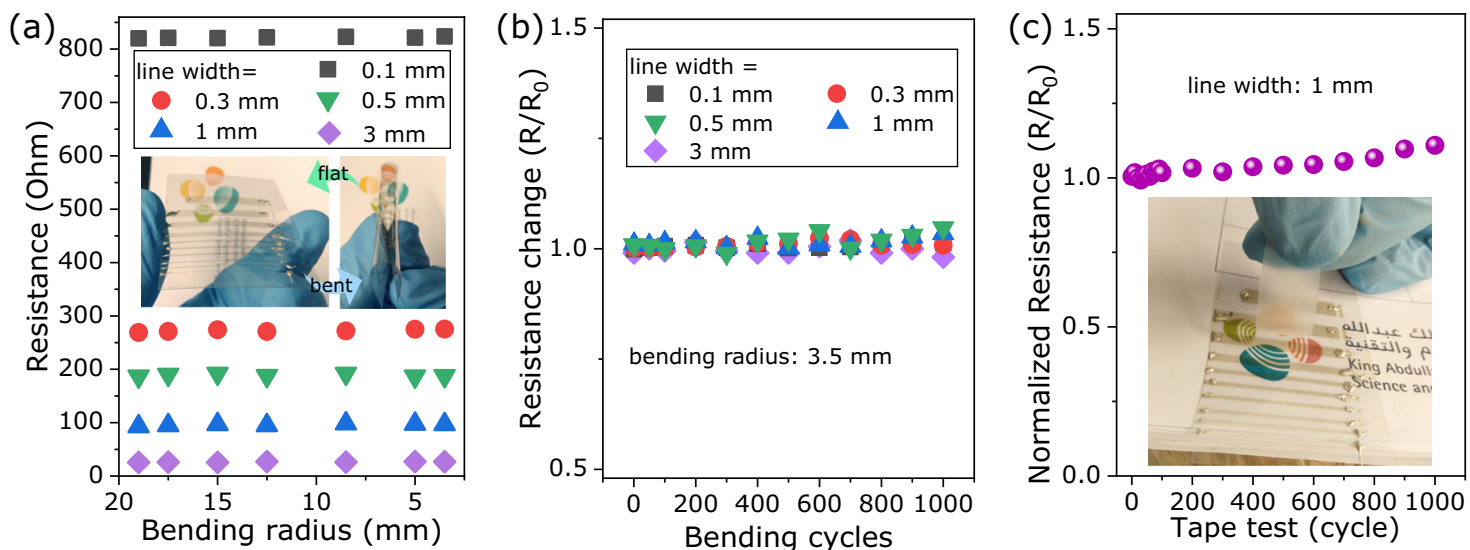

Fig. 3 a The measured resistance as a function of bending radius from $18 \mathrm{~mm}$ to $3.5 \mathrm{~mm}$. The inset photographs show the bending motion of our line patterns. $\mathbf{b}$ The normalized resistance plotted as a function of bending cycles for the patterned Ag NW lines with various line widths. c The normalized resistance of the patterned Ag NW line (width: $1 \mathrm{~mm}$ ) before and after coating with PVA as a protective layer. The photograph displays the adhesion test with scotch tape on Ag NW lines with PVA layer.
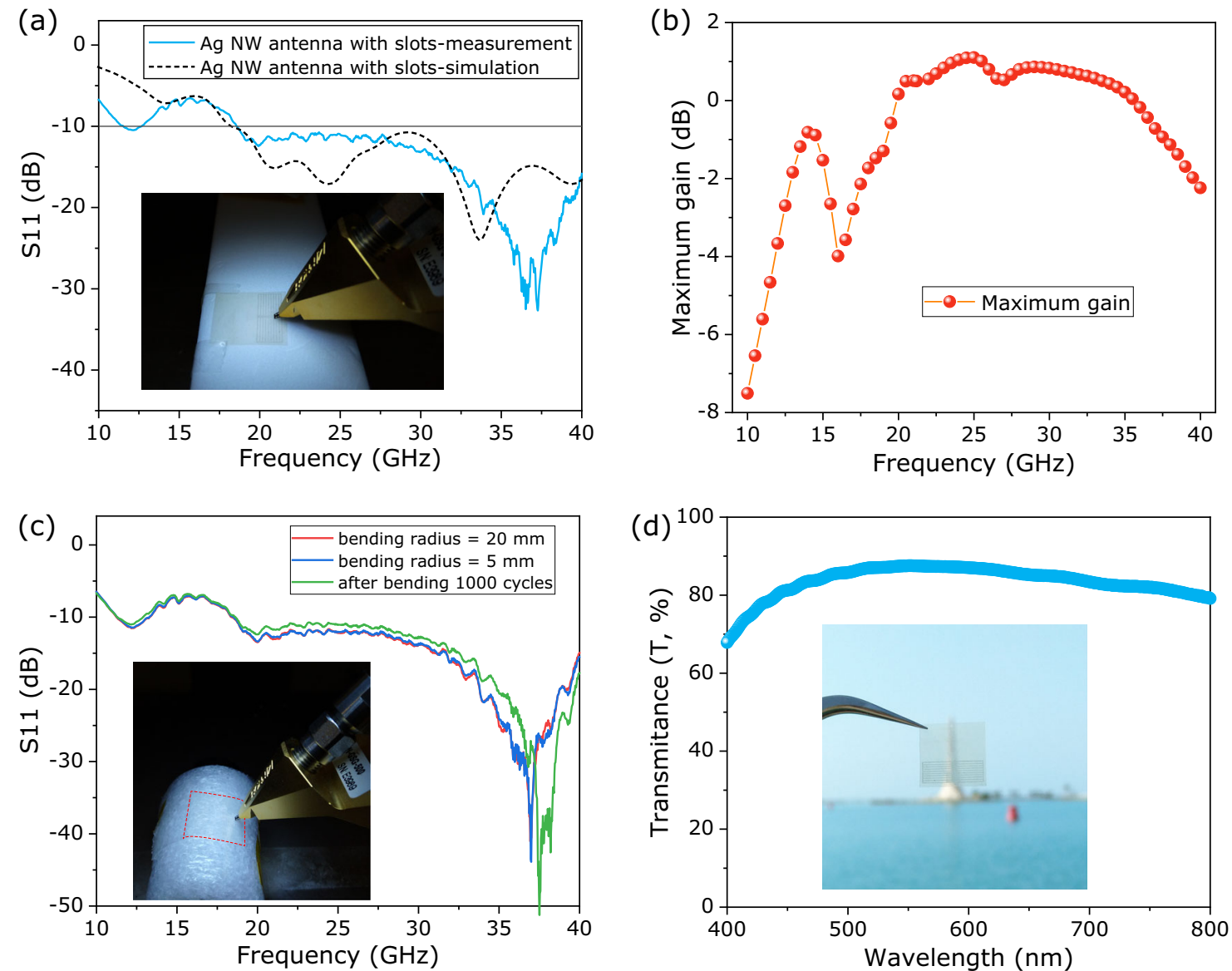

Fig. 4 a The measured and simulated reflection coefficient $\left(S_{11}\right)$ of the fabricated antenna. The inset photograph is a RF coplanar probe used for the high-frequency characterizations. b The simulated antenna gain from 10 to $40 \mathrm{GHz}$. c The $S_{11}$ of the antenna under bending test with different bending radii and multi bending cycles. The flexible antenna demonstrates excellent stability even after 1000 cycles of bending with a bending radius of $3.5 \mathrm{~mm}$. The optical image inset depicts the antenna under test, which is placed on a curved foam surface. $\mathbf{d}$ The transmittance spectra of the fabricated antenna in the wavelength of $400-800 \mathrm{~nm}$. The digital image inset displays the antenna in front of Beacon in the KAUST campus.

To further highlight the cost-effective and time-effective advantages of our coat-and-print method, we demonstrated the patterning of Ag NWs in diverse shapes. Figures 2a and S4 display the patterned Ag NW-based dots (diameter in $60 \mu \mathrm{m}$ ), lines (width in $60 \mu \mathrm{m}$ and spacing in $80 \mu \mathrm{m}$ ), cross lines (width in $60 \mu \mathrm{m}$ ), and squares, circles, and complex drawings with clear edges and boundaries. Essentially, the coat-and-print method is accessible to arbitrary patterns by designing the digital files in commercial software, such as AutoCAD and CorelDraw, or the pattern editor built into the printer software. Notably, the thickness and 
transparency of these patterns are controlled by the concentration of the Ag NW dispersion and coating parameters, and the spatial resolution can be guaranteed by the inkjet printing.

Then, we focused on the basic line patterns and explored how the line parameters affect the patterns' overall electrical conductivity. One important parameter in the coat-and-print method is the printing passes of the PMMA layer: the width of the patterned lines only fluctuated between $86 \mu \mathrm{m}$ and $90 \mu \mathrm{m}$ as the number of printing passes increased (Fig. S5) while the resultant thickness was significantly influenced (Fig. 2b). Specifically, the thickness of the Ag NW lines increased from $\sim 130 \mathrm{~nm}$ to $\sim 420 \mathrm{~nm}$ and became stable $(\sim 420 \mathrm{~nm})$ after three printing passes. This is because that PMMA layers obtained by one or two printing passes were too thin, and thus could not provide sufficient protection for Ag NWs. As a result, some Ag NWs were removed during the follow up wiping process. In contrast, three printing passes were enough to print protective layers to pattern the Ag NWs. In Fig. 2c, we illustrated that the line width were easily controlled by the drop spacing. A drop spacing in the inkjet printer means the deposition distance of two droplets. When the drop spacing is larger, the distance between two deposited ink droplets increases, which results in a line with narrower width. For example, the patterned line width decreased from $180 \mu \mathrm{m}$ to $49 \mu \mathrm{m}$ when the drop spacing increased from $10 \mu \mathrm{m}$ to $50 \mu \mathrm{m}$. SEM images (inset of Fig. $2 c$ ) reveal the line width variation. It can also be seen that the widths of these lines are quite uniform. We then investigated the detailed electrical performance of the patterned lines with line widths ranging from $\sim 0.1 \mathrm{~mm}$ to $\sim 4 \mathrm{~mm}$, and lengths ranging from $5 \mathrm{~mm}$ to $30 \mathrm{~mm}$. Figure $2 \mathrm{~d}$ plots the measured resistance of the Ag NW lines where error bars are created by three time measurements on three separate samples with the same coating and printing parameters. We found that the resistance increases linearly with the line length for all samples, implying the uniformity of the patterned lines using our proposed patterning technique. Quantitatively, sheet resistance $\left(R_{s}\right)$ of the printed lines was calculated according to the measured resistance $(R)$, line width $(w)$ and line length $(I)$ through $R_{s}=R w / l$. The calculated $R_{s}$ for samples with various line widths is displayed in Fig. 2e. The sheet resistance was relatively stable (fluctuating between $2.8 \mathrm{Ohm} / \mathrm{sq}$ to $3.3 \mathrm{Ohm} / \mathrm{sq}$ ) regardless of the line width. These results again prove the uniformity of the patterned Ag NWs, which is particularly essential for the reliability of Ag NW-based electrical devices. We then characterized the electrical conductivity $(\sigma)$ of the patterned $\mathrm{Ag}$ NW lines using line width as a variable by calculating $\sigma=1 /\left(t R_{s}\right)$, where $t$ is the thickness of Ag NW lines. As Fig. $2 f$ illustrates, for the patterned lines with different line widths, the electrical conductivity had a small range $(7-8 \mathrm{kS} / \mathrm{cm})$, again demonstrating the excellent repeatability of the proposed patterning technique.

Having characterized the excellent electrical conductivity and uniformity of the patterned Ag NW lines, we then evaluated the electrical/mechanical stability. The patterned Ag NW lines were firstly subjected to cyclic bending with different radii of curvature and measured their resistance change. Figure 3a demonstrates that the resistance of the patterned $\mathrm{Ag}$ NW lines with various widths can nearly remain constant under a bending radius as low as $3.5 \mathrm{~mm}$. Such durability is further highlighted in Fig. 3b where only slight changes in the resistance are observed for patterned lines after 1000 bending cycles. The pristine Ag NW lines are easy to fail in conductivity after performing tape test (Fig. 3c). To further improve their resistance to wear and friction, $0.5 \%$ polyvinyl alcohol (PVA) solution (water: ethanol=1:1, weight ratio) was spin-coated on the surface of the patterned Ag NW lines. Consequently, the resistance of the patterned line was largely maintained (the relative increase is $~ 20 \%$ ) after 1000 tapepeeling tests, as depicted in Fig. 3c.

The mechanical stability of our patterned Ag NWs, combined with their excellent conductivity and transparency, makes them particularly attractive for flexible electronic devices. To demonstrate the potential applications of the proposed coat-and-print technique for patterning $\mathrm{Ag} \mathrm{NWs}$, a flexible, transparent monopole antenna for the applications of $\mathrm{mm}$-wave, including $5 \mathrm{G}$ communication was fabricated and characterized. Figure $\mathrm{S} 6$ displays the geometric parameters of the proposed radio frequency (RF) antenna and the 3D model in the electromagnetic simulation software (CST Microwave Studio). We used a triangular monopole antenna with slots (width: $150 \mu \mathrm{m}$ ) and a slotted ground layer (to improve transparency), sandwiched between a low-loss dielectric substrate $(\tan \delta=0.0044$ at $24 \mathrm{GHz}$ ) with a thickness of $50 \mu \mathrm{m}$. The total size of the antenna is $12 \times 12 \mathrm{~mm}^{2}$. Figure $4 a$ presents the simulated (dashed line) and measured (solid line) reflection coefficient $\left(S_{11}\right)$ of the monopole antenna, where we found a match in the range of 17.9 to $40 \mathrm{GHz}$. The $-10 \mathrm{~dB}$ bandwidth covers the whole measurement range, indicating an ultra-wide bandwidth, which is beneficial for the applications of $5 \mathrm{G}$ wireless communication. To further evaluate the radiation performance of the fabricated Ag NW monopole antenna, the realized antenna gain was simulated and is dipicted in Fig. 4b. The Ag NW antenna had a reasonable gain, varying from -1.7 to $1.2 \mathrm{dBi}$ from 18 to $40 \mathrm{GHz}$, though the gain was lower than the same antenna made by copper with a conductivity of $5.8 \times 10^{7} \mathrm{~S} / \mathrm{m}$ and a maximum gain of $3.5 \mathrm{dBi}$ (Fig. S7 in Supporting Information).

We conclude our work by highlighting the robustly mechanical flexibility and excellent optical transparency of the fabricated antenna. As displayed in Fig. $4 d$, the measured $S_{11}$ is almost constant under the bending radii of $20 \mathrm{~mm}$ and $5 \mathrm{~mm}$. To further prove the robustness of the monopole antenna under deformation, repetitive bending cycles with the radius of $3.5 \mathrm{~mm}$ were performed, and the reflection coefficient of the antenna was recorded after 1000 cycles. Figure 4c confirms that the $-10 \mathrm{~dB}$ bandwidth performed robustly over a range of $22 \mathrm{GHz}$ without any significant degradations, despite the $S_{11}$ slightly shifting. This result implies that the antenna has an excellent ability to maintain conductive pathways under cyclic deformations. Furthermore, the transmittance of the monopole antenna is presented in Fig. $4 d$, with a flexible Ag NW antenna proves its strong optical transparency with more than $70 \%$ transmittance over the entire visible wavelength range $(400-800 \mathrm{~nm})$. Transmittance at the wavelength of $550 \mathrm{~nm}$ is $86.8 \%$, which is higher than the reported transparent antennas, such as $75 \%$ for micro-metal mesh conductive film-based antenna, ${ }^{56} 80 \%$ for Ag-coated polyesterbased antenna, ${ }^{57}$ and $85 \%$ for graphene-based antenna. ${ }^{58}$

\section{DISCUSSION}

In this paper, we presented a reliable, low-cost coat-and-print technique to pattern Ag NWs with the assistance of inkjet printing. Attributed to the thin and long Ag NWs synthesized in this study, the Ag NW films exhibited excellent conductivity and transmittance compared to the printed transparent electrodes in the literature. Various shapes with different sizes were successfully patterned on rigid glass and flexible substrates with large area and high precision. As a potential application of the proposed patterning approach, flexible and transparent RF antenna was demonstrated and experimentally verified. The antenna had an ultra-wide bandwidth ( $22 \mathrm{GHz}$ of the $-10 \mathrm{~dB}$ bandwidth), reasonable antenna gain (maximum gain: $1.2 \mathrm{~dB}$ ), excellent robustness (more than 1000 bending cycles with a radius of $3.5 \mathrm{~mm}$ ), and high optical transparency (more than $80 \%$ transmittance over the visible range). We believe that the proposed coat-and-print technique can enable fabrication of reliable, precise Ag NW patterns in time-effective and cost-effective ways, and can be extended to other nanomaterials, such as nanoparticles and copper nanowires. 


\section{METHODS}

Synthesis and purification of Ag NWs

The Ag NWs with ultrahigh aspect ratios were synthesized using a modified polyol reduction process. First, $\mathrm{Ag}$ nitrate $(0.12 \mathrm{M}, 10 \mathrm{~mL})$, polyvinyl pyrrolidone (PVP, $60 \mathrm{mM}, 60 \mathrm{~mL}$ ), and cetrimonium bromide (CTAB, $1 \mathrm{~mL}$ ) were prepared in ethylene glycol at room temperature as three separate solutions. In a typical synthesis procedure, the CTAB solution was added to the PVP solution with vigorous stirring for $1 \mathrm{~min}$. Then, the mixed solution was transferred to a reactor preheated to $160^{\circ} \mathrm{C}$. After gently stirring for $30 \mathrm{~min}$, the Ag nitrate solution was injected dropwise into the mixed solution at a jetting rate of $1 \mathrm{~mL} / \mathrm{min}$. Another $60 \mathrm{~min}$ were needed to complete the reaction. Afterward, two-times volume of acetone was added to the cooled dispersion to precipitate the Ag NWs. The product was re-dispersed into water and washed with acetone three times until the supernatant was clear. Then, the product was washed another two times with ethanol to replace the solvent, and redispersed into ethanol with concentrations of $1.5 \mathrm{mg} / \mathrm{mL}, 0.75 \mathrm{mg} / \mathrm{mL}$, and $0.5 \mathrm{mg} / \mathrm{mL}$. Finally, the dispersion was centrifuged at low speed $(\sim 600 \mathrm{rpm}$ for $1 \mathrm{~min}$ ) to obtain well-dispersed Ag NW dispersion by pipetting the supernatant.

\section{Preparation of conductive Ag NW films}

Four-inch glass with a thickness of $1 \mathrm{~mm}, 125-\mu \mathrm{m}$-thick PET and $50-\mu \mathrm{m}$ thick Kapton were used as substrates in the experiment. First, the substrates were carefully cleaned with deionized water and ethanol in the sonication bath. Then they were dried with nitrogen. The obtained Ag NW dispersion was deposited on the cleaned substrates by drop coating or spin coating. The thickness of the Ag layer was determined by the volume and concentration of the Ag NW dispersion and the coating cycles.

\section{Patterning procedures of Ag NWs}

The PMMA powder with a molar ratio of 120,000 was dissolved in a mixed solvent containing anisole and dimethyl sulfoxide (weight ratio of 4:1) to form a $5 \mathrm{wt} \%$ solution, which is ideal for inkjet printing. Afterward, $1.5 \mathrm{~mL}$ of the freshly prepared PMMA was filtered through a $0.2 \mu \mathrm{m}$ nylon filter and then injected into the cartridge. A commercial piezoelectric inkjet nozzle (Dimatix) with a diameter of $21 \mu \mathrm{m}$ and a drop volume of $10 \mathrm{pL}$ was used to inkjet the PMMA solution onto prepared Ag NW films. The temperature of platen was set at $45^{\circ} \mathrm{C}$ to guarantee the uniform spreading of PMMA drops on Ag NW films. Different patterns were designed either through the Dimatix pattern editor or the commercial software Coredraw and then directly printed. After depositing the PMMA protective layer, the sample was placed into an oven at $110^{\circ} \mathrm{C}$ for $5 \mathrm{~min}$ to evaporate the solvents. Next, wet tissue was used to wipe the excess Ag NWs. Finally, the sample was immersed in acetone for $5 \mathrm{~min}$ to dissolve PMMA, followed by drying naturally to complete the patterning of Ag NWs.

\section{Simulation and fabrication of the RF antenna}

Before the fabrication, the antenna structures were optimized and the optimal parameters were simulated in CST Microwave Studio to verify the performance. The monopole antenna was simulated through a thin surface impedance layer with a thickness of $500 \mathrm{~nm}$ and a conductivity of $7 \times 10^{5}$ $\mathrm{S} / \mathrm{m}$. A $50-\mu \mathrm{m}$-thick layer with a dielectric constant of 2.6 and loss tangent of 0.0044 at $24 \mathrm{GHz}$ was used as the dielectric substrate. The source was molded as a discrete $50-0 h m$ port.

To fabricate the monopole antenna, Ag NW dispersion was coated on a clean Preflex substrate (thickness: $0.05 \mathrm{~mm}$ ), dried naturally, and patterned using the method described above. Then, the ground layer was patterned with the same method. Finally, the fabricated antenna was baked in an oven at $100^{\circ} \mathrm{C}$ for $10 \mathrm{~min}$.

\section{Characterization}

The SEM images of the synthesized Ag NWs and the patterned Ag NW films were taken using a scanning electron microscope (FEl NovaNano FEG-SEM 630) after sputtering $3 \mathrm{~nm}$ Ir (Quorum Q150TS) to increase conductivity. The crystallinity of the Ag NWs was examined using XRD (Bruker D2 PHASER) in the range of $30-80^{\circ}$ with a scanning intercept of 0.12 degree/s. The UV-vis absorption spectrum of the Ag NW dispersion and prepared Ag NW films were obtained using a UV-vis spectrophotometer (Thermo Evolution 600). The sheet resistances of the Ag NW films were measured using a four-point probe system (CMT-SR2000N). A vector network analyzer (Agilent N5225A) was used to obtain $S_{11}$ of the fabricated antenna.

\section{DATA AVAILABILITY}

The datasets generated during and/or analyzed during this study are available from the corresponding author on reasonable request.

Received: 9 August 2019; Accepted: 8 November 2019; Published online: 10 December 2019

\section{REFERENCES}

1. Hecht, D. S., Hu, L. \& Irvin, G. Emerging transparent electrodes based on thin films of carbon nanotubes, graphene, and metallic nanostructures. Adv. Mater. 23, 1482-1513 (2011).

2. Krantz, J., Richter, M., Spallek, S., Spiecker, E. \& Brabec, C. J. Solution-processed metallic nanowire electrodes as indium tin oxide replacement for thin-film solar cells. Adv. Funct. Mater. 21, 4784-4787 (2011).

3. Song, M. et al. Highly efficient and bendable organic solar cells with solutionprocessed silver nanowire electrodes. Adv. Funct. Mater. 23, 4177-4184 (2013).

4. Jung, E. et al. Roll-to-roll preparation of silver-nanowire transparent electrode and its application to large-area organic light-emitting diodes. Org. Electron. 41, 190-197 (2017).

5. Yao, S., Cui, J., Cui, Z. \& Zhu, Y. Soft electrothermal actuators using silver nanowire heaters. Nanoscale 9, 3797-3805 (2017).

6. Lee, J.-Y., Connor, S. T., Cui, Y. \& Peumans, P. Solution-processed metal nanowire mesh transparent electrodes. Nano Lett. 8, 689-692 (2008).

7. Park, J. H. et al. Flexible and transparent metallic grid electrodes prepared by evaporative assembly. ACS Appl. Mater. Interfaces 6, 12380-12387 (2014).

8. Qiu, T. et al. Hydrogen reduced graphene oxide/metal grid hybrid film: towards high performance transparent conductive electrode for flexible electrochromic devices. Carbon 81, 232-238 (2015).

9. Schneider, J. et al. Electrohydrodynamic nanodrip printing of high aspect ratio metal grid transparent electrodes. Adv. Funct. Mater. 26, 833-840 (2016).

10. Hong, S. et al. Nonvacuum, maskless fabrication of a flexible metal grid transparent conductor by low-temperature selective laser sintering of nanoparticle ink. ACS Nano 7, 5024-5031 (2013).

11. Vosgueritchian, M., Lipomi, D. J. \& Bao, Z. Highly conductive and transparent PEDOT:PSS films with a fluorosurfactant for stretchable and flexible transparent electrodes. Adv. Fuctional Mater. 22, 421-428 (2012).

12. Li, P., Sun, K. \& Ouyang, J. Stretchable and conductive polymer films prepared by solution blending. ACS Appl. Mater. Interfaces 7, 18415-18423 (2015).

13. Ding, S. et al. One-step fabrication of stretchable copper nanowire conductors by a fast photonic sintering technique and its application in wearable devices. ACS Appl. Mater. Interfaces 8, 6190-6199 (2016).

14. Song, C. H., Han, C. J., Ju, B. K. \& Kim, J. W. Photoenhanced patterning of metal nanowire networks for fabrication of ultraflexible transparent devices. ACS Appl. Mater. Interfaces 8, 480-489 (2016).

15. Park, J. H. et al. Flash-induced self-limited plasmonic welding of silver nanowire network for transparent flexible energy harvester. Adv. Mater. 29, 1603473 (2017).

16. Bae, S. et al. Roll-to-roll production of 30-inch graphene films for transparent electrodes. Nat. Nanotechnol. 5, 574 (2010).

17. Jang, H. et al. Graphene-based flexible and stretchable electronics. Adv. Mater. 28, 4184-4202 (2016).

18. Ning, J. et al. A Facile reduction method for roll-to-roll production of high performance graphene-based transparent conductive films. Adv. Mater. 29, 1605028 (2017).

19. Dai, Z. et al. Interface-governed deformation of nanobubbles and nanotents formed by two-dimensional materials. Phys. Rev. Lett. 121, 266101 (2018).

20. Dai, Z., Liu, L. \& Zhang, Z. Strain engineering of 2D materials: issues and opportunities at the interface. Adv. Mater. 31, 1805417 (2019).

21. Kim, K. S. et al. Large-scale pattern growth of graphene films for stretchable transparent electrodes. Nature 457, 706-710 (2009).

22. Kholmanov, I. N. et al. Optical, electrical, and electromechanical properties of hybrid graphene/carbon nanotube films. Adv. Mater. 27, 3053-3059 (2015).

23. $\mathrm{Wu}, \mathrm{Z}$. et al. Transparent, conductive carbon nanotube films. Science $\mathbf{3 0 5}$, 1273-1276 (2004).

24. Lipomi, D. J. et al. Skin-like pressure and strain sensors based on transparent elastic films of carbon nanotubes. Nat. Nanotechnol. 6, 788-792 (2011).

25. Jiu, J. et al. Facile synthesis of very-long silver nanowires for transparent electrodes. J. Mater. Chem. A 2, 6326-6330 (2014).

26. Celle, C. et al. Highly flexible transparent film heaters based on random networks of silver nanowires. Nano Res. 5, 427-433 (2012). 
27. Choi, S. J., Kim, S. J., Jang, J. S., Lee, J. H. \& Kim, I. D. Silver nanowire embedded colorless polyimide heater for wearable chemical sensors: improved reversible reaction kinetics of optically reduced graphene oxide. Small 12, 5826-5835 (2016).

28. Jung, J. et al. Highly stretchable and transparent electromagnetic interference shielding film based on silver nanowire percolation network for wearable electronics applications. ACS Appl. Mater. Interfaces 9, 44609-44616 (2017).

29. Hu, L., Kim, H. S., Lee, J.-Y., Peumans, P. \& Cui, Y. Scalable coating and properties of transparent, flexible, silver nanowire electrodes. ACS Nano 4, 2955-2963 (2010).

30. Sun, Y., Gates, B., Mayers, B. \& Xia, Y. Crystalline silver nanowires by soft solution processing. Nano Lett. 2, 165-168 (2002).

31. Lee, J. H., Lee, P., Lee, D., Lee, S. S. \& Ko, S. H. Large-scale synthesis and characterization of very long silver nanowires via successive multistep. Growth Cryst. Growth Des. 12, 5598-5605 (2012).

32. Zhang, Y., Guo, J., Xu, D., Sun, Y. \& Yan, F. One-pot synthesis and purification of ultralong silver nanowires for flexible transparent conductive electrodes. ACS Appl. Mater. Interfaces 9, 25465-25473 (2017).

33. Ran, Y., He, W., Wang, K., Ji, S. \& Ye, C. A one-step route to Ag nanowires with a diameter below $40 \mathrm{~nm}$ and an aspect ratio above 1000. Chem. Commun. 50 14877-14880 (2014).

34. Li, B., Ye, S., Stewart, I. E., Alvarez, S. \& Wiley, B. J. Synthesis and purification of silver nanowires to make conducting films with a transmittance of $99 \%$. Nano Lett. 15, 6722-6726 (2015).

35. Xu, F. et al. Preparation and cold welding of silver nanowire based transparent electrodes with optical transmittances $>90 \%$ and sheet resistances $<10 \mathrm{ohm} / \mathrm{sq}$. J. Colloid Interface Sci. 512, 208-218 (2018).

36. De, S. et al. Silver nanowire networks as flexible, transparent, conducting films: extremely high DC to optical conductivity ratios. ACS Nano 3, 1767-1774 (2009).

37. Madaria, A. R., Kumar, A. \& Zhou, C. Large scale, highly conductive and patterned transparent films of silver nanowires on arbitrary substrates and their application in touch screens. Nanotechnology 22, 245201 (2011).

38. Yao, S. \& Zhu, Y. Wearable multifunctional sensors using printed stretchable conductors made of silver nanowires. Nanoscale 6, 2345-2352 (2014).

39. Liang, J., Tong, K. \& Pei, Q. A water-based silver-nanowire screen-print ink for the fabrication of stretchable conductors and wearable thin-film transistors. Adv. Mater. 28, 5986-5996 (2016).

40. Ko, Y. et al. A simple silver nanowire patterning method based on poly(ethylene glycol) photolithography and its application for soft electronics. Sci. Rep. 7, 2282 (2017).

41. Yoon, S. S. \& Khang, D. Y. Facile patterning of Ag nanowires network by microcontact printing of siloxane. ACS Appl. Mater. Interfaces 8, 23236-23243 (2016).

42. Shin, K. et al. Patterned transparent electrode with a continuous distribution of silver nanowires produced by an etching-free patterning method. Sci. Rep. 7, 40087 (2017)

43. Yoo, B., Kim, Y., Han, C. J., Oh, M. S. \& Kim, J.-W. Recyclable patterning of silver nanowire percolated network for fabrication of flexible transparent electrode. Appl. Surf. Sci. 429, 151-157 (2018).

44. Finn, D. J., Lotya, M. \& Coleman, J. N. Inkjet printing of silver nanowire networks. ACS Appl. Mater. Interfaces 7, 9254-9261 (2015).

45. Maisch, P. et al. Inkjet printed silver nanowire percolation networks as electrodes for highly efficient semitransparent organic solar cells. Org. Electron. 38, 139-143 (2016).

46. Cai, L. et al. Direct printing for additive patterning of silver nanowires for stretchable sensor and display applications. Adv. Mater. Technol. 3, 1700232 (2017).

47. Huang, Q., Al-Milaji, K. N. \& Zhao, H. Inkjet printing of silver nanowires for stretchable heaters. ACS Appl. Nano Mater. 1, 4528-4536 (2018).

48. Abutarboush, H. F., Farooqui, M. F. \& Shamim, A. Inkjet-printed wideband antenna on resin-coated paper substrate for curved wireless devices. IEEE Antennas Wirel. Propag. Lett. 15, 20-23 (2016).

49. Li, J., Naiini, M. M., Vaziri, S., Lemme, M. C. \& Östling, M. Inkjet printing of MoS2. Adv. Funct. Mater. 24, 6524-6531 (2014).

50. Cook, B. S., Shamim, A. \& Tentzeris, M. M. Passive low-cost inkjet-printed smart skin sensor for structural health monitoring. IET Microw. Antennas Propag. 6 1536-1541 (2012).

51. Li, W., Yang, S. \& Shamim, A. Screen printing of silver nanowires: balancing conductivity with transparency while maintaining flexibility and stretchability. $n p j$ Flex. Electron. 3, 13 (2019).
52. Su, W., Cook, B. S., Fang, Y. \& Tentzeris, M. M. Fully inkjet-printed microfluidics: a solution to low-cost rapid three-dimensional microfluidics fabrication with numerous electrical and sensing applications. Sci. Rep. 6, 35111 (2016).

53. Liu, S., Ho, S. \& So, F. Novel patterning method for silver nanowire electrodes for thermal-evaporated organic light emitting diodes. ACS Appl. Mater. Interfaces 8 9268-9274 (2016).

54. Ahn, T. et al. A facile patterning of silver nanowires using a magnetic printing method. Nanotechnology 26, 345301 (2015).

55. Layani-Tzadka, M. E., Tirosh, E. \& Markovich, G. Patterning metal nanowire-based transparent electrodes by seed particle printing. ACS Omega 2, 7584-7592 (2017).

56. Li, Q. L., Cheung, S. W., Wu, D. \& Yuk, T. I. Optically transparent dual-band MIMO antenna using micro-metal mesh conductive film for WLAN system. IEEE Antennas Wirel. Propag. Lett. 16, 920-923 (2017).

57. Malek, M. A., Hakimi, S., Rahim, S. K. A. \& Evizal, A. K. Dual-band CPW-fed transparent antenna for active RFID tags. IEEE Antennas Wirel. Propag. Lett. 14, 919-922 (2015).

58. Grande, M. et al. Optically transparent wideband CVD graphene-based microwave antennas. Appl. Phys. Lett. 112, 251103 (2018).

\section{ACKNOWLEDGEMENTS}

Special thanks to KAUST for funding. Special thanks to Liu Wang and Zhaohe Dai from the University of Texas at Austin for fruitful discussions, and contributions to the writing of this paper.

\section{AUTHOR CONTRIBUTIONS}

All authors contributed to the preparation of this paper. W.L. conceived the idea, fabricated the device, performed the test, and analyzed the data. A.M. designed the antenna. A.S. supervised the research and corrected the paper.

\section{COMPETING INTERESTS}

The authors declare no competing interests.

\section{ADDITIONAL INFORMATION}

Supplementary information is available for this paper at https://doi.org/10.1038/ s41528-019-0063-3.

Correspondence and requests for materials should be addressed to W.L.

Reprints and permission information is available at http://www.nature.com/ reprints

Publisher's note Springer Nature remains neutral with regard to jurisdictional claims in published maps and institutional affiliations.

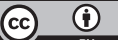

Open Access This article is licensed under a Creative Commons Attribution 4.0 International License, which permits use, sharing, adaptation, distribution and reproduction in any medium or format, as long as you give appropriate credit to the original author(s) and the source, provide a link to the Creative Commons license, and indicate if changes were made. The images or other third party material in this article are included in the article's Creative Commons license, unless indicated otherwise in a credit line to the material. If material is not included in the article's Creative Commons license and your intended use is not permitted by statutory regulation or exceeds the permitted use, you will need to obtain permission directly from the copyright holder. To view a copy of this license, visit http://creativecommons. org/licenses/by/4.0/.

(c) The Author(s) 2019 